CLINICAL ETHICS

\title{
Ethical considerations in presymptomatic testing for variant CJD
}

\author{
R E Duncan, M B Delatycki, S J Collins, A Boyd, C L Masters, J Savulescu
}

See end of article for authors' affiliations

J Med Ethics 2005;31:625-630. doi: 10.1136/jme.2005.011965

....................

Correspondence to: Professor Julian Savulescu, Director, Oxford Uehiro Centre for Practical Ethics, Littlegate House, St Ebbe's Street, Oxford OX1 1PT, UK; julian.savulescu@ philosophy.ox.ac.uk

Received 16February 2005 Accepted for publication 16 February 2005

\begin{abstract}
Variant Creutzfeldt-Jakob disease (vCJD) is a fatal, transmissible, neurodegenerative disorder for which there is currently no effective treatment. VCJD arose from the zoonotic spread of bovine spongiform encephalopathy. There is now compelling evidence for human to human transmission through blood transfusions from presymptomatic carriers and experts are warning that the real epidemic may be yet to come. Imperatives exist for the development of reliable, non-invasive presymptomatic diagnostic tests. Research into such tests is well advanced. In this article the ethical implications of the availability of these tests are elaborated and comparisons drawn with predictive genetic testing for Huntington's disease and screening for HIV. Paramount to considerations is the issue of whom to test, weighing up respect for personal autonomy against obligations to benefit and protect society. A paradigm is proposed similar to that used for HIV screening but with unique features: compulsory testing of all blood/organ donors and individuals undergoing surgery or invasive procedures who have a significant risk of disease transmission.
\end{abstract}

$\mathrm{V}$ ariant Creutzfeldt-Jakob disease (vCJD) was identified in 1996 as distinct from classical forms of CJD (both sporadic and familial). ${ }^{1}$ Cumulative evidence strongly supports that VCJD is zoonotically linked to bovine spongiform encephalopathy (BSE). ${ }^{2-4}$ BSE, CJD, and VCJD are transmissible spongiform encephalopathies (TSEs), a family of invariably fatal neurodegenerative diseases affecting both humans and animals. ${ }^{5}$ They are associated with the accumulation in affected brains of misfolded, protease resistant conformers (PrPres) of a normal host protein known as the prion protein. There is currently no proven prophylaxis or effective treatment for any form of CJD. The molecular structure of the infectious agent is unknown but is referred to as a prion. ${ }^{26}$

Soon after the recognition of vCJD, the tissue distribution of the disease-associated PrPres of the prion protein was shown to be relatively widespread, including in the peripheral lymphoreticular system, heightening concerns of iatrogenic transmission from blood products and surgical procedures. $^{7}$ Adding to these concerns, the transmissible agents causing prion diseases are relatively resistant to the routine methods of decontamination and sterilisation used for medical equipment. ${ }^{8}$ Consequently, imperatives exist for the development of a reliable, non-invasive, presymptomatic or early symptomatic diagnostic test. ${ }^{10-12}$ Research into the development of such tests is well advanced. ${ }^{6}{ }^{13-15}$ In-depth evaluation of the ethical implications of such testing is not.

It had been hoped that the primary wave of vCJD had reached its peak in 2000 when the annual death toll rose to 28 , a figure not reached since. ${ }^{16}$ Two critical findings now negate such optimism. The recent realisation of secondary vCJD transmissions through blood products from presymptomatic donors has raised new fears and reinforced exigencies regarding the development of presymptomatic tests. ${ }^{17-19}$ It is also no longer assumed that vCJD affects only individuals who are homozygous for methionine at codon 129 of the prion protein gene (approximately 33\% of the population), leading experts to wonder if the real epidemic is in fact ahead. ${ }^{18} 2021$

There is an urgent need to consider the ethical implications of the provision of a screening presymptomatic diagnostic test for vCJD. This is crucial for four reasons: (1) vCJD is likely to be transmissible through invasive surgery; (2) sterilisation against vCJD requires more stringent measures; (3) recent data suggest that the number of people potentially incubating $\mathrm{VCJD}$ may be much greater than previously thought; and (4) a presymptomatic or early symptomatic diagnostic test is likely to be available in the near future. Acknowledging each of these facts, we provide an ethical analysis of four possible protocols for test provision, focusing on the need to balance personal autonomy with an obligation to benefit society as a whole. Finally we recommend what we believe to be the best protocol should a presymptomatic test become available.

\section{BRIEF HISTORY}

BSE was first recognised in the UK in 1986. ${ }^{21}$ Cows suffering from BSE show symptoms of anxiety, restlessness, and aggressive behaviour: "mad cow disease". ${ }^{22}$ In 1988 a UK working party recommended the compulsory slaughter of all animals showing symptoms of BSE. ${ }^{23}$ In 1996 vCJD was first described. ${ }^{1}$ That same year, after advice from the Spongiform Encephalopathy Advisory Committee, the UK government announced that there was a probable link between BSE and vCJD. $^{24}$ In 1997 the BSE Inquiry was set up, delivering its report in $2000 .^{23}$

The most common theory on the origins of BSE holds that it was originally transmitted to cows from a sheep prion disease, scrapie, due to cattle feed being supplemented with sheep products. ${ }^{25}$ It is thought that vCJD then arose in humans as a result of exposure to BSE, most likely through contaminated food. ${ }^{10} 26$ The clinical features of vCJD are marked by both neurological and psychiatric disturbances. Individuals often present with anxiety, insomnia, and withdrawal. ${ }^{5}$ Additional clinical features may include delusions, aggression, and auditory and visual hallucinations. ${ }^{27}$ As the disease progresses, neurological signs such as slurred speech, involuntary movements, and cognitive impairments become

Abbreviations: BSE, bovine spongiform encephalopathy; HD, Huntington's disease; PrPres, protease resistant conformers; TSEs, transmissible spongiform encephalopathies; vCJD, variant CreutzfeldtJakob disease 
evident. ${ }^{5}$ The average age of onset is 29 years and the duration of the disease ranges from 9 to 35 months. ${ }^{27}$

Detailed diagnostic criteria for vCJD exist, predicated on the condition representing a neuropsychiatric disorder. ${ }^{28}$ The criteria include early psychiatric symptoms, persistent sensory symptoms, ataxia, myoclonus or chorea or dystonia, and dementia. ${ }^{28}$ Electroencephalography and magnetic resonance imaging are important in diagnostic evaluation, ${ }^{28}$ and tonsil biopsy is also useful for achieving a specific diagnosis of vCJD. $^{29}$

Less than two decades after the first "mad cow", as of December 6, 2004, it is estimated that 147 people have died from vCJD, with an additional five suspected to be living with the disease. ${ }^{16}$ The final vCJD case numbers from primary and secondary transmissions will not be clear for some time but it is obvious that a large percentage of the British population have an elevated risk of developing this disease.

\section{TRANSMISSION OF vCJD IS POSSIBLE THROUGH INVASIVE SURGERY}

TSEs are known to be transmissible both within and between species via the inoculation of infected tissue or dietary exposure to prions. ${ }^{10}$ The first evidence for the transmissibility of human prion diseases was from kuru, a disease discovered in the late 1950s in Papua New Guinea. Transmission of kuru was through cannibalism. ${ }^{10}$ More recent evidence for the transmission of human prion diseases comes from the 267 documented cases of iatrogenic CJD. ${ }^{11}$ These transmission events occurred owing to accidental exposure to prions through medical procedures or treatments. The most common forms of iatrogenic transmission have involved dura mater grafts and growth hormone treatment, but others have included corneal transplants and neurosurgery. ${ }^{11}$ Concerns about the transmissibility of vCJD are heightened by the fact that the tissue distribution of PrPres in VCJD has been shown to be more widespread than in other forms of CJD; specifically, the lymphoreticular system is involved in VCJD. Thus surgical procedures posing potential risks for transmission include tonsillectomy, appendicectomy, and lymph node and gastrointestinal biopsy. ${ }^{27}$ PrPres have been found in the appendix 2 years prior to the onset of symptoms, reinforcing fears of transmission from preclinically affected individuals. ${ }^{15}$

It is generally considered that invasive surgery poses a higher risk for vCJD transmission because the introduction of prions directly into tissues is generally a more efficient route than oral exposure. ${ }^{30}$ Furthermore, same-species transmission of TSEs is also generally more efficient than betweenspecies transmission. ${ }^{30}$

\section{THERE IS NO METHOD PROVEN TO STERILISE AGAINST vCJD WITH CERTAINTY}

Prions, the infectious agents in TSEs, are more resistant to conventional sterilisation and decontamination procedures. ${ }^{5}$ This resistance to classic decontamination methods, combined with knowledge of iatrogenic transmission, poses a serious challenge for public health. ${ }^{9}$ Published guidelines recommend the employment of single-use, disposable instruments. It is also recommended that potentially contaminated devices be incinerated after they are removed from circulation. ${ }^{31}$ Prions have been shown to retain infectivity even after incineration or after being subjected to high autoclave temperatures. ${ }^{31}$

In a case at the Middlesbrough General Hospital, UK, $£ 90000$ worth of surgical instruments had to be withdrawn after the diagnosis of CJD in a patient who had previously undergone a brain biopsy. ${ }^{32}$ The Department of Health subsequently recommended that all surgical instruments used for brain biopsy of non-focal lesions be quarantined until a definitive diagnosis has been obtained. ${ }^{33}$ At this time, the only secure way to guard against the transmission of vCJD through invasive surgery involving high risk tissues or organs is to dispose of medical equipment.

\section{THE vCJD EPIDEMIC MAY EXPAND FURTHER}

The annual death toll from vCJD has been falling since its peak of 28 in 2000. In 2004 the number of deaths dropped to just eight. ${ }^{16}$ Although initial estimates of the total case numbers of vCJD were alarming, they have been revised downwards to a few hundred, creating hope that the worst was over. ${ }^{34}$ However, recent findings have given rise to uncertainties.

There is now compelling evidence for human to human transmission of vCJD through blood transfusions from presymptomatic donors. It has been reported that a recipient of a labile blood component developed symptoms of vCJD approximately 6.5 years after receiving the transfusion. ${ }^{17}$ The donor of the red blood cells developed symptoms of vCJD 3.5 years after the donation, raising the likelihood that the infection was transmitted through the transfusion. A second case has been reported in the UK involving a donor who later went on to develop vCJD. The recipient of this blood transfusion died of causes unrelated to VCJD, but postmortem examination revealed the presence of PrPres in the spleen. ${ }^{18}$

In 2004 up to 4000 individuals were sent letters informing them that they may be at increased risk of carrying vCJD because they had received blood products donated by people who subsequently developed the disease. All recipients were advised to tell their doctors and dentists in order to reduce the possibility of secondary transmission. ${ }^{35}$

Additional fears of a future "tail" or late expansion of the epidemic come from the discovery that vCJD is no longer assumed to occur only in individuals who are homozygous for methionine at codon 129 of the prion protein gene. ${ }^{18} 20$ The patient believed to have contracted vCJD through a blood transfusion was found to be heterozygous at codon 129 at postmortem. ${ }^{18}$ The fear is that those who are homozygous for methionine at codon 129 have shorter incubation periods for vCJD, and those who are heterozygous are not immune to infection, but simply have longer incubation times. ${ }^{13}$ This would imply a further group of people who are currently infected but still asymptomatic, thus posing additional risks for secondary transmission. ${ }^{36}$ Individuals who are homozygous at codon 129 are overrepresented in all forms of sporadic and acquired human TSE and heterozygosity has been shown to correlate with longer incubation periods for growth hormone related CJD and kuru. ${ }^{15}$

Further cause for concern comes from a study of anonymised appendix and tonsil samples, which assessed the presence of PrPres in these tissues. ${ }^{20}$ An analysis of nearly 13000 samples found PrPres in three. If these figures are extrapolated to the population of the UK it would indicate that almost 4000 people aged between 10 and 30 years may be asymptomatically harbouring the prion proteins that cause vCJD. However, only one of the three samples resembled the patterns of accumulation usually seen in vCJD. A more recent study of tonsillectomy specimens failed to find a positive case of disease associated prion proteins in 2000 samples, so more research is needed before definitive conclusions can be drawn. ${ }^{37}$

\section{DEVELOPMENT OF A PRESYMPTOMATIC TEST FOR vCJD}

A reliable, non-invasive, presymptomatic or early symptomatic diagnostic test for vCJD could greatly decrease the potential for iatrogenic transmission in humans but is yet to become available. ${ }^{10-12}$ Many laboratory based approaches are 
being developed for the screening of vCJD using blood samples and will continue to be refined in the future. ${ }^{15}$ Technical aspects of the development of such tests have been detailed, as have methods for validation of test results, although the lack of appropriate positive control samples may hamper the latter. ${ }^{6}{ }^{12}$ Minimal criteria for the sensitivity and specificity of such testing, the circumstances under which tests should be implemented, and the surrounding ethical issues have been the subject of a recent conference. ${ }^{15}$ It is predicted that future screening of blood donors for VCJD may include an initial rapid blood screening test followed by a more rigorous confirmatory test and then a genetic test. ${ }^{15}$

Although it is currently unresolved whether presymptomatically infected persons will invariably manifest illness, these individuals may still pose covert secondary transmission risks in the health care setting. Thus, despite concerns relating to the sensitivity and specificity of any test adopted and the need for the establishment of independent verification methods, the development of a reliable, practicable, presymptomatic diagnostic test would evoke a range of significant ethical concerns.

\section{ETHICAL IMPLICATIONS MUST BE RIGOROUSLY ANALYSED}

The difficulties that may arise in deciding upon a protocol to employ, should presymptomatic testing for vCJD become available, have been acknowledged. ${ }^{30}$ However, a detailed examination of possible protocols and a thorough analysis of the benefits and harms are yet to be undertaken. There is an urgent need to consider these issues prior to the availability of such a test. While the public health benefits of offering such a test are significant, so too is the risk of harm to individuals if testing is administered incorrectly.

\section{COMPARISONS WITH PREDICTIVE GENETIC TESTING FOR HUNTINGTON'S DISEASE}

Huntington's disease (HD) is an autosomal dominant neurodegenerative disorder. It has a mean age of onset of 37 years and no effective treatment is available. On average, HD results in death 15 years after symptom onset. ${ }^{38}$ Presymptomatic genetic testing for HD has been available since 1986. This is generally undertaken in the setting of a formalised protocol whereby, prior to genotyping, those at risk have the opportunity to explore issues around what a positive result and a negative result may mean for them and their family. ${ }^{39}$ An exemplary protocol is outlined in guidelines developed by the International Huntington Association and the World Federation of Neurology. ${ }^{40}$

The majority of those at risk of developing HD choose not to have presymptomatic testing ${ }^{41}$ and guidelines advise against testing anyone under the age of 18 years. ${ }^{40}$ In a study of the outcomes of presymptomatic testing for HD it was found that $2 \%$ of individuals who received a positive result for a causative HD mutation experienced a catastrophic event (defined as committing suicide, attempting suicide, or requiring psychiatric hospitalisation).$^{42}$ This rate of catastrophic events has been interpreted as relatively low, and attributed to the success of the counselling protocol. ${ }^{43}$ In addition to catastrophic events, the literature also reports significant levels of personal stress and social dislocation, including the breakdown of family and social support networks. ${ }^{44}$ It is thought that individuals who ultimately choose to have predictive testing through an informed consent process may be a self-selected group and therefore less likely to suffer major negative consequences when compared with situations in which genotyping is performed unwittingly in persons or against their will. ${ }^{45}$

Common to both HD and VCJD is the fact that a positive test result would lead to individuals being informed they have a high likelihood of developing an invariably fatal disease, currently without treatment. Thus a positive result for vCJD may have similar implications to positive presymptomatic genotyping for HD.

\section{POTENTIAL HARMS AND BENEFITS OF PRESYMPTOMATIC TESTING FOR VCJD}

Using predictive genetic testing for $\mathrm{HD}$ as a paradigm for presymptomatic testing for vCJD, several harmful outcomes are possible, which could include: increased anxiety; depression; guilt; the burden of carrying such knowledge with constant vigilance for the onset of symptoms; discrimination in the workplace or when obtaining insurance; damage to self-esteem; and stigmatisation.

There are two key differences between HD and vCJD that may lead to an increased potential for harm when testing for vCJD. First, the number of people who would be eligible for vCJD testing would most likely be much greater than those eligible for testing for HD. The presentation of a large number of persons for testing may exceed available resources, militating against adequate individual counselling. This scenario could result in higher levels of catastrophic events compared with the rates reported in predictive testing for HD. Secondly, HD is not infectious whereas vCJD is. Consequently, presymptomatic genetic testing for HD will be of no direct benefit to society, while determining whether or not a person carries vCJD could potentially be of considerable public health benefit in the health care setting. There is therefore the possibility of coercion to undertake testing exerted by external authorities such as government agencies, with consequent infringement of personal autonomy. Restricted access to invasive medical procedures is yet another potential harm given the transmissibility of vCJD.

The clearest support for presymptomatic testing for vCJD is a possible benefit to public health. Testing would allow the exclusion of blood and tissue/organ donations from asymptomatic carriers. Preoperative and preprocedure screening could be systematically undertaken as part of infection control measures for patients undergoing a range of surgical and other invasive procedures involving higher risk tissues. Personal benefits from presymptomatic testing are also likely. A presymptomatic test for vCJD could be used to reassure the "worried well". Additional benefits include the opportunity for psychological adjustment, the opportunity for making realistic future plans (including end of life decisions, decisions about financial planning, and decisions about the timing of having children), and a reduced time between symptom onset and diagnosis.

\section{THE ETHICALLY BEST PROTOCOL FOR PRESYMPTOMATIC TESTING OF VCJD}

When exploring possible protocols, two key decisions are paramount. The first decision is about consent: should the test be voluntary or compulsory? The second decision is about whom to test: should all people be tested or only selected populations (for example, individuals undergoing medical procedures with an increased risk for transmission-defined from this point as a risk procedure). Based on these two decisions, there are at least four possible protocols: (1) all persons wanting to be tested can be tested; (2) all persons undergoing a risk procedure who want to be tested can be tested; (3) all persons must be tested; and (4) all persons undergoing a risk procedure must be tested. Table 1 summarises these four protocols. ${ }^{18}$

The principal competing issues when deciding upon a protocol for the provision of presymptomatic testing for vCJD are respect for personal autonomy and the promotion of public health. If we consider the four protocols in terms of personal autonomy, it is clear that the protocol maximising 
Table 1 Four protocols for presymptomatic testing for vCJD

\begin{tabular}{lll}
\hline & Universal testing & Selective testing \\
\hline Voluntary testing & $\begin{array}{l}\text { Protocol 1: all persons who want to be } \\
\text { tested can be tested }\end{array}$ & $\begin{array}{l}\text { Protocol 2: all persons undergoing a medical } \\
\text { procedure posing a potentially higher risk of } \\
\text { transmission can be tested }\end{array}$ \\
Compulsory testing & Protocol 3: all persons must be tested & $\begin{array}{l}\text { Protocol 4: all persons undergoing a medical } \\
\text { procedure posing a potentially higher risk of } \\
\text { transmission must be tested }\end{array}$ \\
\hline
\end{tabular}

personal autonomy is the one allowing every person who wants to undergo presymptomatic testing for vCJD to do so (protocol 1). Table 2 presents an analysis of the four protocols with respect to personal autonomy.

If we consider the four protocols in terms of the promotion of public health, it is clear that the protocol maximising public health and safety is the one enforcing compulsory testing of all individuals undergoing risk procedures (protocol 4). Table 3 presents an analysis of the four protocols with respect to the promotion of public health.

We argue that the ethically most appropriate protocol is the one that offers the best compromise between individual autonomy and the greatest benefit to society. That is, a protocol where all blood/organ donors and individuals undergoing surgery and invasive procedures with a significant theoretical risk of disease transmission undergo compulsory testing but where any other individual desiring a test also has access to one. This is a combination of protocols 1 and 4 above.

Given the possibilities for discrimination in the insurance and employment sectors, we recommend this testing protocol be subject to legislation that bans the use of presymptomatic test results by insurance companies or employers. This is in line with recommendations made by the Human Genetics Commission of the UK. In their report titled Inside information, they recommend that "the government consider in detail the possible need for separate UK legislation to prevent genetic discrimination and that this evaluation form part of a longterm policy review on the use of personal genetic information in insurance and employment." ${ }^{\prime 46}$ We make similar recommendations here.

\section{COMPARISONS WITH TESTING FOR HIV}

Should a cost effective presymptomatic test for vCJD become available, our ethically preferred protocol is similar to the current approach for HIV testing. Anyone who desires to undergo a test for HIV can do so. However, if individuals donate blood (posing a risk to others), their blood is screened for HIV and, if a positive result is returned, they are informed of their HIV status. Also included in the current HIV protocol is compulsory before and after test counselling. We strongly recommend that the same level of counselling be made compulsory for presymptomatic vCJD testing.

The major difference between HIV testing and testing for vCJD is that, if individuals do not want to know their HIV status, they can simply choose not to donate blood. However, some surgery, including neurosurgery, for which individuals will need to undergo screening for vCJD, may be essential for survival. Thus, it is not realistic to assume that, if individuals do not want to be tested for vCJD, they can safely choose not

Table 2 An analysis of the four protocols with respect to personal autonomy

\begin{tabular}{ll}
\hline Protocol & Ability to promote personal autonomy \\
\hline $\begin{array}{l}\text { Protocol 1: All persons who want to be tested } \\
\text { can be tested }\end{array}$ & $\begin{array}{l}\text { Maximises personal autonomy by allowing all persons to } \\
\text { decide for themselves }\end{array}$ \\
$\begin{array}{l}\text { Protocol 2: All persons undergoing a medical } \\
\text { procedure involving a high risk of transmission }\end{array}$ & $\begin{array}{l}\text { Maintains personal autonomy but limits it to a subset of the } \\
\text { population }\end{array}$ \\
$\begin{array}{l}\text { can be tested } \\
\text { Protocol 3: All persons must be tested }\end{array}$ & $\begin{array}{l}\text { Fails to respect personal autonomy by removing choice for } \\
\text { all persons }\end{array}$ \\
$\begin{array}{l}\text { Protocol 4: All persons undergoing a medical } \\
\text { procedure involving a high risk of transmission } \\
\text { must be tested }\end{array}$ & $\begin{array}{l}\text { Fails to respect personal autonomy by removing choice for } \\
\text { a subset of the population }\end{array}$ \\
\hline
\end{tabular}

Table 3 An analysis of the four protocols with respect to the promotion of public health

\begin{tabular}{ll}
\hline Protocol & Ability to promote public health \\
\hline $\begin{array}{l}\text { Protocol 1: All persons who want to be tested } \\
\text { can be tested }\end{array}$ & $\begin{array}{l}\text { May help to promote public health but only if people who } \\
\text { are undergoing higher risk procedures choose to be tested; } \\
\text { many may not }\end{array}$ \\
$\begin{array}{l}\text { Protocol 2: All persons undergoing a medical } \\
\text { procedure involving a higher risk of transmission } \\
\text { can be tested }\end{array}$ & $\begin{array}{l}\text { May help to promote public health but only if people } \\
\text { choose to be tested; many may not }\end{array}$ \\
$\begin{array}{ll}\text { Protocol 3: All persons must be tested } & \text { Maximum effect on public health by reducing the possibility } \\
\text { of transmission but at a large economic cost }\end{array}$ \\
$\begin{array}{l}\text { Protocol 4: All persons undergoing a medical } \\
\text { procedure involving a higher risk of transmission } \\
\text { must be tested }\end{array}$ & $\begin{array}{l}\text { Maximum effect on public health by reducing the possibility } \\
\text { of transmission and is more cost effective than protocol } 3\end{array}$ \\
\hline
\end{tabular}




\section{Summary of main points}

1. Variant Creutzfeldt-Jakob disease (VCJD) arose from the zoonotic spread of bovine spongiform encephalopathy (BSE).

2. Prophylaxis for VCJD does not exist and carriers may be asymptomatic.

3. Human to human transmission of VCJD has most likely occurred through blood transfusions and VCJD remains difficult to sterilise against using conventional methods.

4. Imperatives exist for the development of a presymptomatic diagnostic test for vCJD and the ethical implications of test provision require consideration.

5. When a presymptomatic test for VCJD becomes available, we propose compulsory testing of all blood/organ donors and individuals undergoing surgery and invasive procedures that have a significant risk of disease transmission, and the provision of testing to any individuals voluntarily requesting it.

to undergo surgery. One alternative may be to offer people the option of non-disclosure of test results. This would allow them to remain unaware of their vCJD status, while still benefiting the health of the public. Furthermore, it is important to remember that hospitals are able relatively easily to guard against HIV transmission via the application of universal precautionary measures for all individuals undergoing medical procedures. This is not currently the situation with regard to vCJD. The disease agent causing vCJD is not easily destroyed by routine sterilisation methods and requires a higher level of disinfection practice and precautions to limit the risk of secondary transmission. ${ }^{96}$

\section{HEALTH ECONOMIC CONSIDERATIONS}

Omitted from our discussion and deliberations regarding the ethical issues of presymptomatic testing for vCJD has been health economic considerations. If we are to view public health in a comprehensive (and realistic) manner, the costs associated with each testing protocol must also be considered. From a health economics perspective, the optimum protocol, using society as a whole as the point of reference, will be the protocol that minimises transmission, while remaining cost effective. Some of the health economic concerns associated with presymptomatic testing for vCJD may be the cost of counselling, the cost of looking after people who are infected with VCJD, the cost of disposing of surgical instruments, the cost of performing the test (depending on whether the Government or the individual pays), and the number of catastrophic events.

\section{CONCLUSION}

When a practicable, presymptomatic test for vCJD becomes available prior to the development of any effective prophylaxis or treatment, an ethically based screening protocol is suggested. We recommend compulsory testing of all blood/ organ donors and individuals undergoing surgery and invasive procedures with a significant risk of disease transmission, and also the provision of testing to any individual who voluntarily requests it. Counselling and support services similar to those established in the HIV model are strongly recommended. Testing should also be subject to legislation that bans the discriminatory use of presymptomatic test results by insurance companies or employers. The preferred protocol is akin to that used for current HIV screening and would benefit public health by minimising the risk of covert transmission events.

\section{ACKNOWLEDGEMENTS}

We would like to thank Professor John Collinge for his helpful comments on the manuscript.

\section{Authors' affiliations}

R E Duncan, Murdoch Children's Research Institute and the Bruce Lefroy Centre for Genetic Health Research, Royal Children's Hospital, Parkville, Australia; Department of Paediatrics and Centre for the Study of Health and Society, Department of Public Health, University of Melbourne, Australia

M B Delatycki, Murdoch Children's Research Institute, the Bruce Lefroy Centre for Genetic Health Research and Genetic Health Services

Victoria, Royal Children's Hospital, Parkville, Australia; Department of Paediatrics, University of Melbourne, Australia

S J Collins, A Boyd, C L Masters, Australian National Creutzfeldt-Jakob Disease Registry, Department of Pathology, University of Melbourne, Parkville, Australia

J Savulescu, Murdoch Children's Research Institute, Royal Children's Hospital, Parkville, Australia; Oxford Uehiro Centre for Practical Ethics, University of Oxford, UK

Competing interests: none declared

\section{REFERENCES}

1 Will RG, Ironside JW, Zeidler $M$, et al. A new variant of Creutzfeldt-Jakob disease in the UK. Lancet 1996;347:921-5.

2 Collinge J, Sidle KCL, Meads J, et al. Molecular analysis of prion strain variation and the aetiology of 'new variant' CJD. Nature 1996;383:685-90.

3 Bruce ME, Will RG, Ironside JW et al. Transmissions to mice indicate that 'new variant' CJD is caused by the BSE agent. Nature 1997;389:498-501.

4 Hill AF, Desbruslais $M$, Joiner S, et al. The same prion strain causes VCJD and BSE. Nature 1997;389:448-50.

5 Collins SJ, Lawson VA, Masters CL. Transmissible spongiform encephalopathies. Lancet 2004;363:51-61.

6 Minor PD. Technical aspects of the development and validation of tests for variant Creutzfeldt-Jakob disease in blood transfusion. Vox Sanguinis 2004;86:164-70

7 Wadsworth JDF, Joiner S, Hill AF, et al. Tissue distribution of protease resistant prion protein in variant Creutzfeldt-Jakob disease using a highly sensitive immunoblotting assay. Lancet 2001;358:171-80.

8 Bernoulli C, Siegfreid J, Baumgartner G, et al. Danger of accidental person-toperson transmission of Creutzfeldt-Jakob disease by surgery. Lancet 1977;i:478-9.

9 Fichet G, Comoy E, Duval C, et al. Novel methods for disinfection of prioncontaminated medical devices. Lancet 2004;364:521-6.

10 Trevitt CR, Singh PN. Variant Creutzfeldt-Jakob disease: pathology, epidemiology, and public health implications. Am J Clin Nutr 2003;78(suppl):651S-6S.

11 Brown P, Preece M, Brandel J-P, et al. latrogenic Creutzfeldt-Jakob disease at the millennium. Neurology 2000;55:1075-81.

12 Safar J, Wille $\mathrm{H}$, Itri $\mathrm{V}$, et al. Eight prion strains have PrPSc molecules with different conformations. Nat Med 1998;4:1157-65.

13 Bonn D. Future uncertain for reliable vCJD screening tests. Lancet 2000;356:228.

14 British Broadcasting Corporation. We may have a test for vCJD [BBC News]. 16 March 2004. http://news.bbc.co.uk/go/pr/fr/-/hi/health/ 3517184.stm (accessed 18 Mar 2004)

15 Blajchman MA, Goldman M, Webert KE, et al. Proceedings of a consensus conference: the screening of blood donors for variant CJD. Transfus Med Rev 2004; 18:73-92.

16 The National Creutzfeldt-Jakob Disease Surveillance Unit. CJD figures Update 6 Dec 2004.http://.www.cjd.ed.ac.uk (accessed 8 Dec 2004) [For specific up to date figures, http://www.cjd.ed.ac.uk/figures.htm].

17 Llewelyn CA, Hewitt PE, Knight RSG, et al. Possible transmission of variant Creutzfeldt-Jakob disease by blood transfusion. Lancet 2004;363:417-21.

18 Peden AH, Head MW, Ritchie DL, et al. Preclinical vCJD after blood transfusion in a PRNP codon 129 heterozygous patient. Lancet 2004;364:527-9.

19 Bird SM. Recipients of blood or blood products "at vCJD risk". BMJ 2004;328:118-9.

20 Hilton DA, Ghani AC, Conyers L, et al. Prevalence of lymphoreticular prion protein accumulation in UK tissue samples. J Pathol 2004;203:733-9.

21 Wells GA, Scott AC, Johnson CT, et al. A novel progressive spongiform encephalopathy in cattle. Vet $\operatorname{Rec} 1987 ; 31: 419-20$.

22 Tan L, Williams MA, Khan MK, et al. Risk of transmission of bovine spongiform encephalopathy to humans in the United States: Report of the council of scientific affairs. JAMA 1999;281:2330-9.

23 Lord Phillips of Worth Matravers, Bridgeman J, Ferguson-Smith M. The BSE Inquiry: the inquiry into BSE and variant CJD in the United Kingdom. Oct 2000. http://www.bseenquiry.gov.uk/index.htm (accessed 8 Jan 2005)

24 Almond J, Pattison J. Human BSE. Nature 1997;389:437-8.

25 Brown P. On the origins of BSE [Commentary]. Lancet 1998;352:252-3.

26 Mishra RS, Basu S, Gu Y, et al. Protease-resistant human prion protein and ferritin are cotransported across Caco-2 epithelial cells: implications for 
species barrier in prion uptake from the intestine I Neurosci 2004; $24: 11280-90$.

27 Collinge J. Prion diseases of humans and animals: their causes and molecular basis. Annu Rev Neurosci 2001;24:519-50.

28 Will RG, Zeidler M, Stewart GE, et al. Diagnosis of new variant CreutzfeldtJakob disease. Ann Neurol 2000;47:575-82.

29 Hill AF, Butterworth RJ, Joiner S, et al. Investigation of variant CreutzfeldtJakob disease and other human prion diseases with tonsil biopsy samples. Lancet 1999:353:183-9.

30 Horby P. Variant Creutzfeldt-Jakob disease: an unfolding epidemic of misfolded proteins. J Paediatr Child Health 2002;38:539-42.

31 McDonnell G, Burke P. The challenge of prion decontamination. Clin Infect Dis 2003;36:1152-4.

32 Hunter M. Inquiry into handling of CJD alert welcomed. BMJ 2002;325:1055.

33 Collinge J. Variant Creutzfeldt-Jakob disease, Lancet 1999:354:317-23.

34 Sneath PHA. Estimation of the size of the VCJD epidemic. Antonie can Leeuwenhoek 2004;86:93-103

35 Kmietowicz Z. Patients informed of increased risk of vCJD contact. BM 2004;329:702

36 Spinney L. vCJD epidemic could be first of many, experts warn [News]. Nat Med 2003;9:1096.

37 Frosh A, Smith LC, Jackson CJ, et al. Analysis of 2000 consecutive UK tonsillectomy specimens for disease-related prion protein. Lancet 2004;364:1260-2.
38 Myers R, Marans K, MacDonald M. Huntington's disease. In: Wells R, Warren S, eds. Genetic instabilities and hereditary neurological diseases. San Diego, CA: Academic Press, 1998:301-23.

39 Delatycki MB, Tassicker R. Adult onset neurological disorders: predictive genetic testing. Aust Fam Physician 2001;30:948-52.

40 International Huntington Association and the World Federation of Neurology Research Group on Huntington's Chorea. Guidelines for the molecular genetics predictive test in Huntington's disease. J Med Genet 1994;31:555-9.

41 Binedell J, Soldan JR, Harper PS. Predictive testing for Huntington's disease: predictors of uptake in South Wales. Clin Genet 1998:54:477-88.

42 Almqvist EW, Bloch $M$, Brinkman R, et al. A worldwide assessment of the frequency of suicide, suicide attempts, or psychiatric hospitalisation after predictive testing for Huntington disease. Am J Hum Genet 1999:64:1293-304

43 Bird T. Outrageous fortune: the risk of suicide in genetic testing for Huntington disease. Am J Hum Genet 1999;64:1289-92.

44 Sobel SK, Cowan DB. Impact of genetic testing for Huntington disease on the family system. Am J Med Genet 2000;90:49-59.

45 Meiser B, Dunn S. Psychological effects of genetic testing for Huntington's disease: an update of the literature. West J Med 2001;174:336-40.

46 Human Genetics Commission (UK). Inside information: balancing interests in the use of personal genetic data. 2002. http://www.hgc.gov.uk (accessed 1 Feb 2005).

47 Gregori L, McCombie N, Palmer D, et al. Effectiveness of leucoreduction for removal of infectivity of transmissible spongiform encephalopathies from blood. Lancet 2004;364:529-31.

\section{Notice}

Ethical aspects of the new genetics: what we all need to know

This one day conference and debate is open to all and will take place at the Cheltenham Town Hall on Friday 18 November 2005.

Tickets are $£ 10$ and are available from Gloucestershire Federation of WI's, 2 Brunswick Square, GL1 1UL, tel: 014522396 66; email: liz@gfhi.org.uk. For futher information visit www.gfwi.org.uk

A limited number of free tickets funded by the Institute of Medical Ethics are available to health care students. Apply with staff confirmation of student status by sending a SAE to Maureen Bannatyne, Insitute of Medical Ethics, St Chloe, The Avenue, Old Bussage, Glos GL6 8AT. 